\title{
Monotherapy versus Combination Therapy against Nonbacteremic Carbapenem-resistant Gram-negative Infections: A Retrospective Observational Study
}

\author{
Abdul Ghafur, Vidyalakshmi Devarajan, T. Raja', Jose Easow', M. A. Raja', Sankar Sreenivas', Balasubramaniam Ramakrishnan², S. G. Raman", \\ Dedeepiya Devaprasad ${ }^{3}$, Balaji Venkatachalam ${ }^{3}$, Ramesh Nimmagadda ${ }^{1}$ \\ Departments of Infectious Diseases, ${ }^{1}$ Medical Oncology, ${ }^{2}$ Medical Statistics and ${ }^{3}$ Intensive Care, Apollo Cancer Institute, Chennai, Tamil Nadu, India
}

\section{Abstract}

Background: Superiority of colistin-carbapenem combination therapy (CCCT) over colistin monotherapy (CMT) against carbapenem-resistant Gram-negative bacterial (CRGNB) infections is not conclusively proven. Aim: The aim of the current study was to analyze the effectiveness of both strategies against CRGNB nonbacteremic infections. Design: This was a retrospective observational cohort study. Subjects and Methods: Case record analysis of patients who had CRGNB nonbacteremic infections identified over a period of 4 years (January 2012-December 2015) was done by medical record review at a tertiary care center in India. Statistical Analysis: $P<0.05$ was considered as significant. Multivariate analysis was performed using Cox regression. Results: Out of 153 patients (pneumonia 115, urinary tract infection 17, complicated skin and soft-tissue infection 18, intra-abdominal infection 1, and meningitis 2), 92 patients received CCCT and 61 received CMT. Univariate analysis revealed higher Acute Physiology and Chronic Health Evaluation II (APACHE II) score, pneumonia as the diagnosis, and Klebsiella as the causative organism to be the risk factors for higher 28-day mortality $(P=0.036,0.006,0.016$, respectively). Combination therapy had no significant impact on mortality (odds ratio $[\mathrm{OR}]=0.91,95 \%$ confidence interval $[\mathrm{CI}]=0.327-2.535, P=0.857)$. Multivariate analysis revealed that higher APACHE II score and infection due to Klebsiella were found to be independent risk factors for higher mortality (OR $=3.16$ and 4.9, 95\% CI $=1.34-7.4$ and 2.19-11.2, $P=0.008$ and 0.0001 , respectively). Conclusions: In our retrospective single-center series of CRGNB nonbacteremic infections, CCCT was not superior to CMT. Multicenter large observational studies or prospective randomized clinical trials are the need of the hour.

Keywords: Colistin-based combination therapy, colistin monotherapy, New Delhi metallo-beta-lactamase, extensively drug resistant, Gram-negative bacteria

\section{INTRODUCTION}

Management of infections due to carbapenem-resistant Gram-negative bacteria (CRGNB) is a therapeutic challenge. ${ }^{[1-3]}$ Colistin-based combination therapy (CCT) is a popular strategy employed against these infections. There are multiple studies and systematic reviews supporting this approach ${ }^{[4-10]}$ On the contrary, a meta-analysis of all the available combination studies questioned the effectiveness of combination strategy. ${ }^{[11]}$ A retrospective study of colistin monotherapy (CMT) versus CCT against CRGNB bloodstream infections from our own center revealed equivalence of both approaches, except in a subgroup of neutropenic patients with Enterobacteriaceae infections where CCT arm performed better ${ }^{[12]}$ There are no published data from India comparing CCT and CMT against CRGNB nonbacteremic infections.

\begin{tabular}{|l|l|}
\hline \multicolumn{2}{|c|}{ Access this article online } \\
\hline Quick Response Code: & Website: \\
\hline & www.ijccm.org \\
\cline { 2 - 2 } & \\
\hline
\end{tabular}

The aim of the present study was to analyze the effectiveness of both strategies against CRGNB nonbacteremic infections.

\section{Subjects and Methods}

Retrospective analysis of patients who had carbapenem-resistant Gram-negative (CRGNB) nonbacteremic infections identified over a period of 4 years (January 2012-December 2015) was done by medical record review at a 300 -bedded tertiary care oncology, neurosurgical and orthopedic center in India. The

Address for correspondence: Dr. Abdul Ghafur, Apollo Cancer Institute, Chennai, Tamil Nadu, India. E-mail: drghafur@hotmail.com

This is an open access article distributed under the terms of the Creative Commons Attribution-NonCommercial-ShareAlike 3.0 License, which allows others to remix, tweak, and build upon the work non-commercially, as long as the author is credited and the new creations are licensed under the identical terms.

For reprints contact: reprints@medknow.com

How to cite this article: Ghafur A, Devarajan V, Raja T, Easow J, Raja MA, Sreenivas S, et al. Monotherapy versus combination therapy against nonbacteremic carbapenem-resistant gram-negative infections: A retrospective observational study. Indian J Crit Care Med 2017;21:825-9. 
Institutional Ethics Committee approved this study. As the study design was retrospective observational, informed consent was waived.

Bacterial identification and susceptibility were done using VITEK-2 compact system (bioMérieux, France). Susceptibility results were interpreted according to the Clinical and Laboratory Standards Institute guideline. CRGNB was defined as resistant to imipenem and/or meropenem in vitro. Colistin susceptibility was tested using VITEK-2 compact for all isolates, and minimum inhibitory concentration was calculated for some isolates by $E$-test according to availability. Hospital identification numbers of patients with CRGNB nonbacteremic infections were recovered from hospital digital database, and their medical records were tracked and analyzed.

A patient is considered cured from infection if there is microbiological cure or no additional antibiotic therapy for the targeted infection necessary. Patients were classified as having nonbacteremic CRGNB infection if: (a) there was recovery of a CRGNB isolate from cultures of intra-abdominal wounds, urine, respiratory tract specimens (sputum, endotracheal aspirate, or bronchoalveolar lavage fluid), or other sites; (b) there was no blood culture positivity for CRGNB for the duration of hospitalization; (c) there were clinical signs of infection; and (d) patients were receiving treatment with an antimicrobial regimen that was consistent with the isolate's in vitro susceptibility profile. ${ }^{[13]}$

Data for variables such as age, sex, underlying immunocompromising condition, comorbidities, Intensive Care Unit (ICU) stay, duration of the first isolate from admission, colistin administration within $24 \mathrm{~h}$ of index date of sample collection, colistin dose, duration and cumulative dose, presence of indwelling devices, and prior antibiotic exposure were looked into. ${ }^{[12]}$ Charlson comorbidity index was calculated for all patients. Acute Physiology and Chronic Health Evaluation II (APACHE II) score was calculated for ICU patients. The outcome including 28-day mortality was analyzed. Antimicrobial therapy was considered appropriate if the regimen included antibiotics that were active in vitro. Those patients who received colistin, carbapenem, or tigecycline for $<72 \mathrm{~h}$ were excluded from the study. Neutropenia was defined as a neutrophil count lower than $1000 / \mathrm{mm}^{3}$. Treatment given before obtaining susceptibility results was defined as empirical. An empirical antimicrobial treatment regimen was considered as inadequate if it did not include at least one drug displaying in vitro activity against the CRGNB isolate. Regimens were classified as CMT or CCT depending on whether the patient received colistin alone or any other antibiotics included in the study (carbapenem, aminoglycoside, and tigecycline).

Statistical analysis was performed using SPSS 16.0. $P<0.05$ was considered as statistically significant. ${ }^{[12]}$ Multivariate analysis using Cox regression was performed, incorporating univariate variables with $P<0.05$, for 28 -day mortality. All categorical variables were represented by percentages.
Continuous variables were represented by mean \pm standard deviation. Comparison of categorical variables was done by Chi-square test or Fisher's exact test.

\section{RESULTS}

Out of 153 patients (pneumonia 115, urinary tract infection 17 , complicated skin and soft-tissue infection 18, intra-abdominal infection 1, and meningitis 2), 92 patients received CCT and 61 received CMT. Acinetobacter baumannii was the causative organism in $35 \%$ of the patients, A. baumannii and Klebsiella pneumoniae in 3\%, Escherichia coli in 5\%, K. pneumoniae in 42\%, and Pseudomonas aeruginosa in 15\%. Out of the 92 patients who received CCT, 24 had Acinetobacter and received colistin-carbapenem (ColCarb) combination in 15, colistin-carbapenem-tigecycline (ColCarbTig) in 2, colistin-carbapenem-tigecycline-sulbactam (ColCarbTigS) in 4 , and colistin-carbapenem-sulbactam (ColCarbS) in 3. Two patients in combination arm had mixed infection with carbapenem-resistant Acinetobacter and Klebsiella (both received colistin and carbapenem). Out of the 6 patients with E. coli in CCT arm, 3 had ColCarb and 3 had ColTig. Out of 47 Klebsiella, 28 had ColCarb, 8 ColCarbTig, and 11 ColTig. All the 13 Pseudomonas in the CCT arm received ColCarb combination.

Crude mortality was high in the CCT arm (38\% [35/92]) compared to the monotherapy arm (18\% [11/61]), but CCT arm had sicker patients with more number of patients in ICU and on inotropes $(92.4 \%$ vs. $73.8 \%, P=0.002,37 \%$ vs. $16.4 \%$, $P=0.006$, respectively). Characteristics of patients are shown in Table 1.

Univariate and multivariate Cox regression analyses for all-cause 28-day mortality were done [Table 2]. Univariate analysis revealed that a higher APACHE II score of $>24$ (odds ratio $[\mathrm{OR}]=2.975,95 \%$ confidence interval $[\mathrm{CI}]=1.075-8.235 ; P=0.036)$, pneumonia as the syndromic diagnosis $(\mathrm{OR}=4.465,95 \% \mathrm{CI}=1.543-12.917 ; P=0.006)$, and Klebsiella as the etiological agent $(\mathrm{OR}=3.536,95 \%$ $\mathrm{CI}=1.269-9.856 ; P=0.016)$ were significantly associated with higher 28-day mortality. Colistin administration on the index date $(\mathrm{OR}=1.935,95 \% \mathrm{CI}=0.737-5.078 ; P=0.18)$ and combination therapy $(\mathrm{OR}=0.91,95 \% \mathrm{CI}=0.327-2.535$; $P=0.857)$ did not have any protective effect on the 28-day mortality. Multivariate analysis revealed that high APACHE II score $(\mathrm{OR}=3.16,95 \% \mathrm{CI}=1.34-7.4 ; P=0.0080)$ and Klebsiella as the etiological agent $(\mathrm{OR}=4.9,95 \%$ $\mathrm{CI}=2.19-11.2 ; P=0.0001)$ were independently associated with higher 28-day mortality. As the sample size was not big enough, we did not perform subgroup analyses of various combinations.

\section{Discussion}

In our retrospective study of patients with nonbacteremic carbapenem-resistant Gram-negative bacterial infections, colistin-carbapenem combination therapy (CCCT) was 


\begin{tabular}{|c|c|c|c|c|}
\hline Variables & Categories & Colistin alone $(n=61), n(\%)$ & Combination $(n=92), n(\%)$ & $P$ \\
\hline \multirow[t]{2}{*}{ Sex } & Male & $48(78.7)$ & $70(76.1)$ & 0.708 \\
\hline & Female & $13(21.3)$ & $22(23.9)$ & \\
\hline \multirow[t]{5}{*}{ Diagnosis (site of infection) } & Meningitis & $1(1.6)$ & $1(1.1)$ & 0.352 \\
\hline & Pneumonia & $45(73.8)$ & $70(76.1)$ & \\
\hline & Intra-abdominal infection & 0 & $1(1.1)$ & \\
\hline & UTI & $10(16.4)$ & $7(7.6)$ & \\
\hline & Soft-tissue infection & $5(8.2)$ & $13(14.1)$ & \\
\hline \multirow[t]{5}{*}{ Organism } & Acinetobacter & $23(37.7)$ & $24(26.1)$ & 0.265 \\
\hline & Acinetobacter and Klebsiella & $1(1.6)$ & $2(2.2)$ & \\
\hline & Escherichia coli & $3(4.9)$ & $6(6.5)$ & \\
\hline & Klebsiella & $21(34.4)$ & $47(51.1)$ & \\
\hline & Pseudomonas & $13(21.3)$ & $13(14.1)$ & \\
\hline \multirow[t]{4}{*}{ Underlying condition } & $\mathrm{HO}$ & $1(1.6)$ & $6(6.5)$ & 0.162 \\
\hline & NS & $32(52.5)$ & $35(38.0)$ & \\
\hline & ST & $21(34.4)$ & $33(35.9)$ & \\
\hline & Others & $7(11.5)$ & $18(19.6)$ & \\
\hline Colistin dose & Median & $9(9-9)$ & $9(9-9)$ & 0.139 \\
\hline Colistin duration & Median & $8(5-10.5)$ & $8(4-13)$ & 0.605 \\
\hline Colistin cumulative dose & Mean & $71 \pm 33.8$ & $72 \pm 6.5$ & 0.461 \\
\hline Colistin on index date & Given & $15(24.6)$ & $34(37.0)$ & 0.108 \\
\hline $\mathrm{CCU}$ & Yes & $45(73.8)$ & $85(92.4)$ & 0.002 \\
\hline Surgery & Yes & $41(67.2)$ & $46(50.0)$ & 0.035 \\
\hline Neutropenia & Yes & $5(8.2)$ & $5(5.4)$ & 0.499 \\
\hline Inotropes & Yes & $10(16.4)$ & $34(37.0)$ & 0.006 \\
\hline Mechanical ventilation & Yes & $38(62.3)$ & $76(82.6)$ & 0.005 \\
\hline Lines & Yes & $29(47.5)$ & $52(56.5)$ & 0.276 \\
\hline Foleys & Yes & $42(68.9)$ & $53(57.6)$ & 0.16 \\
\hline \multirow[t]{3}{*}{ Microbiological cure } & Cure & $5(8.2)$ & $8(8.7)$ & 0.52 \\
\hline & Not cure & $16(26.2)$ & $17(18.5)$ & \\
\hline & Not repeated & $40(65.6)$ & $67(72.8)$ & \\
\hline Clinical cure & Cure & $50(82.0)$ & $57(62.0)$ & 0.008 \\
\hline 28-day outcome & Mortality & $11(18.0)$ & $35(38.0)$ & 0.008 \\
\hline
\end{tabular}

UTI: Urinary tract infection; CCU: Critical Care Unit; HO: Haemato-oncology; NS: Neurosurgery; ST: Solid Tumour

Table 2: Univariate and multivariate Cox regression analyses for all-cause 28-day mortality

\begin{tabular}{|c|c|c|c|c|c|c|}
\hline \multicolumn{4}{|c|}{ Logistic regression univariate analysis } & \multicolumn{3}{|c|}{ Multivariate analysis } \\
\hline Risk factors & OR & $95 \% \mathrm{Cl}$ & $P$ & OR & $95 \% \mathrm{Cl}$ & $P$ \\
\hline Age & 2.285 & $0.705-7.404$ & 0.168 & & & \\
\hline Sex & 0.651 & $0.218-1.941$ & 0.441 & & & \\
\hline APACHE score & 2.975 & $1.075-8.235$ & 0.036 & 3.16 & $1.34-7.4$ & 0.008 \\
\hline Charlson index & 1.497 & $0.453-4.948$ & 0.508 & & & 0.419 \\
\hline Pneumonia & 4.465 & $1.543-12.917$ & 0.006 & 1.5 & $0.07-28.6$ & 0.785 \\
\hline Klebsiella & 3.536 & $1.269-9.856$ & 0.016 & 4.9 & $2.19-11.2$ & 0.0001 \\
\hline Pseudomonas & 0.725 & $0.123-4.261$ & 0.722 & & & \\
\hline Colistin on index date & 1.935 & $0.737-5.078$ & 0.18 & & & \\
\hline Colistin monotherapy/combination therapy & 0.91 & $0.327-2.535$ & 0.857 & & & \\
\hline Inotropes & 0.862 & $0.302-2.457$ & 0.781 & & & \\
\hline Mechanical ventilation & 4.138 & $0.949-18.037$ & 0.059 & & & \\
\hline Lines & 1.944 & $0.702-5.377$ & 0.201 & & & \\
\hline Constant & 0.09 & & 0.086 & & & \\
\hline
\end{tabular}

OR: Odds ratio; CI: Confidence interval; APACHE: Acute Physiology and Chronic Health Evaluation

not superior to CMT. Combination therapy arm had higher all-cause 28-day mortality than the monotherapy arm. This was not unexpected in a retrospective study, with more number of sicker patients (in ICU and on inotropes) receiving 
combination therapy. Logistic regression analysis adjusting for the confounders including disease severity did not reveal any protective effect of combination therapy, denoting nonsuperiority of CCCT over CMT.

Carbapenem-resistant Gram-negative bacterial infections result in high morbidity and mortality of immunocompetent as well as immunocompromised patients. Various retrospective cohort studies and a few systematic reviews support the concept of CCT. ${ }^{[4-10]}$ At the same time, the only available meta-analysis on the subject could not demonstrate any superiority of the combination therapy over CMT. ${ }^{[1]}$ A large retrospective study of 661 patients with 447 bloodstream infections (Tumbarello et al.) found CCCT superior to CMT if the carbapenem MIC is $<8 \mathrm{mg} / \mathrm{L} \cdot{ }^{[13]} \mathrm{A}$ recently published large multicenter study did not find colistin-based combination superior to monotherapy, except in a subgroup of patients with high pretreatment probability of death. ${ }^{[14]}$ A retrospective study of 91 patients with CRGNB bloodstream infections, from our own oncology center, found CMT to be as effective as CCCT, except in a subgroup of Enterobacteriaceae infections in neutropenia where the combination therapy arm had better outcome. ${ }^{[12]}$ We could find only two other combination studies from India, both small observational studies with no significant difference in the outcome between the two groups. ${ }^{[15,16]}$ There are no published data from India comparing CCCT and CMT against CRGNB nonbacteremic infections. To the best of our knowledge, this is the first publication on combination therapy in nonbacteremic infections.

High carbapenem Minimum Inhibitory Concentration (MIC) (MIC $>16 \mathrm{mg} / \mathrm{L})$ of most CRGNB isolates in the center (unpublished data), similar to available published data from other Indian centers, could possibly be one of the reasons for the lack of superiority of CCCT with carbapenem in comparison to CMT. ${ }^{[15,17]}$ It is worth noting that even in the Tumbarello study, CCCT was superior only if the carbapenem MIC was $<8 \mathrm{mg} / \mathrm{L}$. ${ }^{[13]}$

Administration of appropriate antibiotic (an antibiotic sensitive against CRGNB) within $24 \mathrm{~h}$ of collection of the index isolate was also not associated with a protective effect in reducing mortality. This finding is similar to most other published studies on treatment of CRGNB infections, including the recently published large multicenter combination study that showed administration of antibiotics within the first 5 days (but not within the first 2 days) resulted in mortality reduction. ${ }^{[6,7,9]}$ We did not analyze the impact of appropriate antibiotic administration after $24 \mathrm{~h}$ (for example, within 2 days and within 5 days of index culture) which could have shown a protective effect of the appropriate antibiotic administration. High APACHE II score and Klebsiella as the etiologic agent were independently associated with higher 28-day all-cause mortality.

The main limitation of our study was the retrospective design and the inherent limitation of single-center data in solving combination therapy conundrum. To prevent the risk for spuriously significant associations between individual treatment regimens and mortality, retrospective observational studies on the monotherapy versus combination therapy may require several hundreds or thousands of patients. Only multicenter studies can achieve compilation of such a large data. Prospective randomized trials could be the ideal solution to the monotherapy versus combination therapy dilemma. We also need to explore the benefits of noncarbapenem-based combinations.

\section{Conclusions}

In our retrospective, single-center series of carbapenem-resistant Gram-negative nonbacteremic infections, the outcome was similar in CCCT and CMT arms. Multicenter large observational studies or prospective randomized clinical trials are the need of the hour. The role of colistin-based combination with noncarbapenem antibiotics also needs to be explored.

\section{Acknowledgment}

We express our gratitude to Mr. Karthik, Ms. Veena, and Mr. Ramakrishnan for assisting us in data collection. The study was carried out with the research grant from AstraZeneca, as an investigator-initiated study. The design or data collection of the study and the content of the paper are in no way influenced by the grant provider.

\section{Financial support and sponsorship}

The study was carried out with the research grant from AstraZeneca pharmaceuticals, as an investigator-initiated study. The design or data collection of the study and the content of the paper are in no way influenced by the grant provider.

\section{Conflicts of interest}

There are no conflicts of interest.

\section{RefEREnCES}

1. Kim YJ, Kim SI, Hong KW, Kim YR, Park YJ, Kang MW, et al. Risk factors for mortality in patients with carbapenem-resistant Acinetobacter baumannii bacteremia: Impact of appropriate antimicrobial therapy. J Korean Med Sci 2012;27:471-5.

2. Andria N, Henig O, Kotler O, Domchenko A, Oren I, Zuckerman T, et al. Mortality burden related to infection with carbapenem-resistant gram-negative bacteria among haematological cancer patients: A retrospective cohort study. J Antimicrob Chemother 2015;70:3146-53.

3. Ghafur A, Mathai D, Muruganathan A, Jayalal JA, Kant R, Chaudhary D, et al. The Chennai declaration: A roadmap to tackle the challenge of antimicrobial resistance. Indian J Cancer 2013;50:71-3.

4. Falagas ME, Lourida P, Poulikakos P, Rafailidis PI, Tansarli GS. Antibiotic treatment of infections due to carbapenem-resistant enterobacteriaceae: Systematic evaluation of the available evidence. Antimicrob Agents Chemother 2014;58:654-63.

5. Tumbarello M, Viale P, Viscoli C, Trecarichi EM, Tumietto F, Marchese A, et al. Predictors of mortality in bloodstream infections caused by Klebsiella pneumoniae carbapenemase-producing K. pneumoniae: Importance of combination therapy. Clin Infect Dis 2012;55:943-50.

6. Qureshi ZA, Paterson DL, Potoski BA, Kilayko MC, Sandovsky G, Sordillo E, et al. Treatment outcome of bacteremia due to KPC-producing Klebsiella pneumoniae: Superiority of combination antimicrobial regimens. Antimicrob Agents Chemother 2012;56:2108-13.

7. Daikos GL, Tsaousi S, Tzouvelekis LS, Anyfantis I, Psichogiou M, Argyropoulou A, et al. Carbapenemase-producing Klebsiella 
pneumoniae bloodstream infections: Lowering mortality by antibiotic combination schemes and the role of carbapenems. Antimicrob Agents Chemother 2014;58:2322-8.

8. Falagas ME, Rafailidis PI, Ioannidou E, Alexiou VG, Matthaiou DK, Karageorgopoulos DE, et al. Colistin therapy for microbiologically documented multidrug-resistant gram-negative bacterial infections: A retrospective cohort study of 258 patients. Int J Antimicrob Agents 2010;35:194-9.

9. Zarkotou O, Pournaras S, Tselioti P, Dragoumanos V, Pitiriga V, Ranellou K, et al. Predictors of mortality in patients with bloodstream infections caused by KPC-producing Klebsiella pneumoniae and impact of appropriate antimicrobial treatment. Clin Microbiol Infect 2011;17:1798-803.

10. Lee GC, Burgess DS. Treatment of Klebsiella pneumoniae carbapenemase (KPC) infections: A review of published case series and case reports. Ann Clin Microbiol Antimicrob 2012;11:32.

11. Paul M, Carmeli Y, Durante-Mangoni E, Mouton JW, Tacconelli E, Theuretzbacher $\mathrm{U}$, et al. Combination therapy for carbapenem-resistant gram-negative bacteria. J Antimicrob Chemother 2014;69:2305-9.

12. Ghafur AK, Vidyalakshmi PR, Kannaian P, Balasubramaniam R. Clinical study of carbapenem sensitive and resistant gram-negative bacteremia in neutropenic and nonneutropenic patients: The first series from India. Indian J Cancer 2014;51:453-5.

13. Tumbarello M, Trecarichi EM, De Rosa FG, Giannella M, Giacobbe DR, Bassetti M, et al. Infections caused by KPC-producing Klebsiella pneumoniae: Differences in therapy and mortality in a multicentre study. J Antimicrob Chemother 2015;70:2133-43.

14. Gutiérrez-Gutiérrez B, Salamanca E, de Cueto M, Hsueh PR, Viale P, Paño-Pardo JR, et al. Effect of appropriate combination therapy on mortality of patients with bloodstream infections due to carbapenemase-producing enterobacteriaceae (INCREMENT): A retrospective cohort study. Lancet Infect Dis 2017;17:726-34.

15. Shah PG, Shah SR. Treatment and outcome of carbapenem-resistant gram-negative bacilli blood-stream infections in a tertiary care hospital. J Assoc Physicians India 2015;63:14-8.

16. Porwal R, Gopalakrishnan R, Rajesh NJ, Ramasubramanian V. Carbapenem resistant gram-negative bacteremia in an Indian Intensive Care Unit: A review of the clinical profile and treatment outcome of 50 patients. Indian J Crit Care Med 2014;18:750-3.

17. Kazi M, Shetty A, Rodrigues C. The carbapenemase menace: Do dual mechanisms code for more resistance? Infect Control Hosp Epidemiol 2015;36:116-7. 